\title{
De la enseñanza de la literatura a la educación literaria
}

\section{Teresa Colomer}

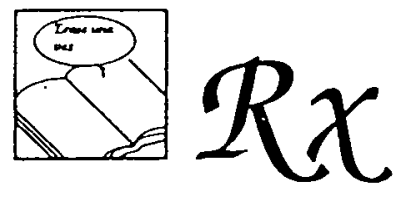

Los cambios que se han producido en el repertorio de materiales lingüisticos que le presenta la cultura al niño ban sido tan grandes como los que se han dado en la comprensión teórica y educativa de lo lingüistico como sistema que debe adquirir y dominar el niño. Estos cambios exigen una actualización de la enseñanza de la lengua y la literatura de modo que siga justificadamente teniendo el papel relevante que antes merecidamente ocupaba, con contenidos, objetivos y tareas distintos, en un mundo cultural también distinto. En este artículo se hace un análisis crítico de ambas situaciones $y$ se propone un programa para la construcción de un saber leer literario en el marco de la enseñanza de la lectoescritura y la lengua.

En el curso de las dos últimas décadas se ha señalado repetidamente (Federicis, 1985) que el modelo tradicional de enseñanza de la literatura en la escuela se halla sumido en una situación de crisis profunda e irreversible. Puede constatarse también cómo, a pesar del tiempo transcurrido, la improcedencia de la antigua concepción no ha sido sustituida aún de forma global y coherente por una nueva propuesta educativa, y cómo, en consecuencia, nos hallamos en la actualidad ante una gran desorientación respecto a la función educativa de la literatura y a su posible programación escolar. Parece indicado pensar que la superación de esta etapa debe provenir de la reflexión educativa sobre los progresos teóricos producidos en las distintas ciencias implicadas en la enseñanza de la literatura, del análisis y valoración de los avances ya producidos de forma intuitiva y fragmentaria en la práctica escolar y de la integración de todos estos elementos en un nuevo marco teórico que permite fundamentar y desarrollar una nueva articulación de objetivos y prácticas educativas. A la luz de los conocimientos actuales puede afirmarse 
que este nuevo marco deberá reemplazar definitivamente la concepción de una enseñanza de la literatura por la de una educación literaria, entendida esta última, tal como viene definiéndose en las nuevas corrientes didácticas (Coveri, 1986; Lugarini, 1985), -como la adquisición de una competencia lectora específica que requiere del reconocimiento de una determinada conformación lingüística y del conocimiento de las convenciones que regulan la relación entre el lector y este equipo de texto en el acto concreto de su lectura.

\section{LOS AVANCES YA PRODUCIDOS EN LA SITUACION ACTUAL}

Si bien, hasta el momento, las consecuencias educativas de los progresos realizados en los distintos campos teóricos se han producido más como negación o disolución del modelo precedente que como articulación de una verdadera formación literaria, puede constatarse la generalización escolar de algunos presupuestos y prácticas educativas que sin duda señalan el avance hacia una nueva propuesta y que pueden esquematizarse en los apartados siguientes:

\section{Dónde se lee. La nueva situación curricular}

La delimitación del texto literario como una de las posibilidades de la lengua escrita a partir de los avances de la lingüística de los años sesenta ha establecido una nueva relación entre educación lingüística y educación literaria. Así, la división tradicional entre un predominio casi absoluto del texto literario en el área de lengua y el uso sin instrucción linguística alguna del texto informativo en las otras áreas de enseñanza parece evolucionar hacia el estudio escolar de una amplia gama de textos a través de un planteamiento más acorde con la tipología establecida por la lingüística textual (Adam, 1987, 1988; Combettes, 1988) y más capaces de responder a la necesidad educativa de mejorar la comprensión y producción de todo tipo de textos.

Es cierto, sin embargo, que la saludable pérdida de hegemonía del texto literario lleva aparejada una cierta perplejidad sobre su nueva función. Si bien en la enseñanza media la existencia de un espacio docente claramente delimitado por los planes de estudio ha preservado su enseñanza en la escuela primaria, y lógicamente de forma especial en el ciclo superior de EGB, su suerte ha sido mucho más variable.

No se trata, verdaderamente, de un problema de presencia del texto literario, puesto que en coda la escuela primaria resulta habitual la lectura de un amplio abanico de narraciones, descripciones, diálogos y poemas más o menos fragmentados. La dificultad radica en establecer los objetivos adecuados para las actividades que deben realizarse a partir de esas lecturas, ya que es fácil observar que en muy escasa medida se proponen iniciar y desarrollar la educación literaria de los alumnos, y, de hecho, la utilización más generalizada del texto literario consiste en servir de introducción para las actividades lingüísticas. Efectivamente, el análisis de los libros de texto más frecuentes confirma que la mayor parte de las propuestas toman el texto como mera provisión de palabras o frases para tratar contenidos léxico-semánticos, gramaticales o de producción escrita con escasa relación con los aspectos literarios del texto leído. 
Ciertamente, antes de abordar estos contenidos tan diversificados, suelen proponerse una serie de acrividades de comprensión del texto que permitirían una real profundización en la competencia lectora, y específicamente en la literaria, si estuvieran planteados según los conocimientos actuales sobre el proceso de lectura, entendido como construcción de una representación mental coherente, y sobre las características del texto literario. Pero recientes investigaciones (Solé, 1987, por ejemplo, en nuestro país) han vuelto a reafirmar el carácter fundamentalmente evaluativo de este tipo de actividades encaminadas a la comprensión literal y que toman habitualmente la forma de un repertorio de preguntas. Estas preguntas acostumbran a reclamar un recuerdo inmediato de pequeños detalles secundarios y frecuentemente atañen a informaciones obtenidas según el desarrollo cronológico de la acción. El tipo de respuesta así obtenido es una simple verificación, escueta y fácilmente localizable en el texto, aún en el caso que el lector no lo haya comprendido, ya que no exige ningún tipo de elaboración personal ni se refiere a la comprensión global del texto.

\section{Qué se lee. La ampliación del corpus}

Si la lengua literaria ya no es vista como la cima de las posibilidades de expresión de una lengua y entra en crisis su presentación modélica e histórica a través de los autores clásicos, se hace acuciante la necesidad escolar de hallar textos literarios más cercanos a los intereses y a la capacidad comprensiva de los alumnos. Para responder a esta demanda se produce una extensión del corpus literario utilizado en dos sentidos principales.

La literatura de tradición oral, ya presente en alguna medida en los primeros niveles de enseñanza, amplía su presencia en ellos y aparece en los ciclos posteriores. A sus virtudes de enlace con fórmulas conocidas por los niños desde antes de su escolarización se une su explotación en la fase de aprendizaje de la lengua escrita e incluso su reivindicación de patrimonio cultural en peligro por la urbanización de la sociedad o, en algunos casos, por las décadas de marginación cultural de las lenguas minoritarias. La apreciación de estas cualidades por parte de los enseñantes ha propiciado una extensión casi abusiva de este tipo de literatura a toda la enseñanza obligatoria con el riesgo de limitar en un nuevo sentido la experiencia literaria propuesta a los alumnos.

La literatura infantil y juvenil, por otra parte, ha penetrado con tal fuerza en el ciclo educativo que a menudo constituye una alternativa práctica real frente al desconcierto escolar sobre la programación literaria. Si durante mucho tiempo fue desatendida la necesidad de su presencia en la escuela o se la arrinconó a una utilización casi extraescolar en la biblioteca del centro, en estos momentos se halla muy generalizado su uso en la formación lectora a través de actividades de incitación a la lectura y de creación de hábitos lectores, ya que no hay duda de que esta literatura resulta especialmente idónea para la familiarización con el texto y, tal como Wells (1986) ha señalado, para la realización de importantes aprendizajes lectores y literarios desde edades muy tempranas, aprendizajes que se refieren tanto a conocimiento implícito de las convenciones básicas sobre el tiempo literario o la división de la acción en secuencias, como a la exper:mentación del placer verbal, la 


\section{4}

identificación con los personajes o el diálogo con la propia experiencia.

Sin embargo, su utilización escolar presenta también peligros contrapuestos: Por una parte, su función continua inscribiéndose mayoritariamente en el terreno estricto de la motivación lectora, aún en los casos en que su presencia ha desbancado la lectura de cualquier otro texto literario. Ello conlleva la práctica inexistencia de una programación de actividades de análisis y comprensión que pudiera hacer progresivamente explícitos los conocimientos literarios y ayudar de este modo al lector en su tarea de interpretar el texto con una competencia cada vez mayor. Por otra parte, asistimos, en cambio, a la proliferación de guías de trabajo y lectura de estas obras de ficción, de tal forma que estos textos se convierten en el nuevo punto de partida para el mismo tipo de rutinas escolares anteriormente señaladas, con el agravante de anular la capacidad motivadora de un corpus literario que, en consonancia con la voz de alerta lanzada por Battelheim y Zelan (1981), se presenta como real y no creado artificialmente para su uso escolar.

\section{Cómo se lee. El acceso al texto}

Sin duda la primera consecuencia de los cambios combinados de la lingüística y la teoría literaria estructuralistas de los años sesenta y de las bases psicopedagógicas de la enseñanza de la lectura fue el abandono de la transmisión memorística de la información sobre el patrimonio literario y su sustitución por el acceso directo del lector al texto. El comentario de texto se convirtió a continuación en la actividad didáctica por excelencia en los niveles educativos donde existía la programación histórica. El profesor continuaba siendo, de todos modos, el poseedor de la información y el encargado de glosar el texto, o bien desde nuevas perspectivas sociológicas, psicológicas, etc., o bien desde la propia construcción textual a partir de un complejo análisis que durante un tiempo hizo concebir a los enseñantes la esperanza de haber encontrado una base teórica de la propia disciplina que les pudiera proporcionar técnicas científicas equivalentes a la solidez referencial de los avances lingüísticos.

Desde entonces, e incluso desde la relación no resuelta entre teoría literaria y enseñanza de la literatura, la necesidad del contacto entre el lector y el texto no ha hecho más que afianzarse como un hecho indiscutible que se ha nutrido, en los últimos años, de la atención concedida al lector desde diversas disciplinas, desde la psicología cognitiva a la teoría de la recepción literaria (Littérature et enseignement, la perspective du lecteur, 1988). Este contacto entre el texto y el lector se produce actualmente en la escuela a través de diversos niveles de fragmentación de la obra y de distintas formas de lectura. La situación más usual es la combinación de la lectura de fragmentos o textos breves ya señalada, la lectura individual de obras completas, generalmente para la creación de hábitos lectores no sujetos a actividades regladas, y la lectura colectiva de una o varias obras.

Este último tipo de lectura resulta especialmente interesante al proporcionar el referente común necesario para ejercitar la competencia literaria con la ayuda del profesor y del resto de lectores en los aspectos de construcción global de la obra, aspectos imposibles de analizar en fragmentos aislados. La anticipación de los diversos elementos narrativos, la selección de indicios 
relevantes, la integración de la información sobre las nuevas acciones y conductas de los personajes y, sobre todo, el análisis del propio autocontrol sobre la coherencia interpretativa hallan en la lectura integral colectiva un espacio privilegiado para el aprendizaje interactivo de las estrategias de lectura, estrategias que el lector podrá trasladar posteriormente a otras obras de lectura individual, de acuerdo con el método defendido por Collins y Smith (1980) para el aprendizaje progresivamente autónomo de la comprensión lectora. Lamentablemente, esta actividad didáctica se traduce mayoritariamente en un trabajo centrado en los breves fragmentos leídos en cada clase sin que se establezca su enlace global con la obra entera. Se repiten así, por lo tanto, el mismo tipo de actividades realizadas a partir de la lectura de textos breves, con la única salvedad del interés y conocimiento de una obra entera por parte de los alumnos.

\section{Leer y escribir. La producción de textos literarios}

La propuesta de construir nuevos textos literarios a imitación de los propuestos no es ninguna novedad en la enseñanza literaria, ni tampoco lo es la producción de textos que versan sobre su forma y significado tales como resúmenes, comentarios y paráfrasis. En realidad puede afirmarse que estas prácticas tradicionales se han desvirtuado y disminuído ante el impacto de la perplejidad ante la enseñanza de la literatura. En los años setenta, sin embargo, irrumpirán en este campo otro tipo de actividades de escritura que parecían corresponderse con la difusión de nuevos objetivos de acceso activo al placer literario, tanto en su recepción como en su emisión, a través de la desacralización de la obra literaria. Las propuestas de Petitjean $(1982,1984)$ constituyen ejemplos, especialmente rigurosos y sugerentes, de este tipo de actividades que, a menudo bajo la forma de talleres literarios, y muy masivamente en la escuela primaria a partir de la divulgación de la obra de G. Rodari, tomaron el relevo de la tradicional redacción escolar. De modo simplificado podemos dividir las técnicàs expresivas más utilizadas en tres grandes grupos:

A) Técnicas referidas a la manipulación de las obras con introducción de cambios en la estructura narrativa, en el punto de vista o en las coordenadasespacio-temporales, y a su reelaboración, -a menudo paródica o de inversión de sentido.

B) Técnicas de producción de textos originales con la aplicación de estímulos para la creación tomados con frecuencia de las prácticas literarias vanguardistas: asociación libre, juegos de palabras, extrañamientos, etc.

C) Técnicas de creación textual a partir de modelos retóricos diversos: modalidades expresivas, cambios de género literario o descubrimiento/adopción de una estructura textual previamente fijada.

El principal problema de estas nuevas actividades radica en $s u$ falta de vertebración en el curriculum escolar, en su realización como un espacio de creación placentera y al margen de cualquier saber reflexivamente organizado. Efectivamente, su práctica no responde a objetivos claramente definidos ni está sujeta a programación alguna, por lo cual es imposible la evaluación de resultados, la selección de las ayudas necesarias y la conciencia por parte del 
alumno de criterios objetivos con los que valorar su progreso personal en el dominio de la expresión escrita.

La realización de las actividades de los dos primeros grupos señalados, se circunscribe básicamente al maxco del estímulo para la creación y adolece de la falta de un desarrollo mínimamente riguroso para el proceso posterior. La creación es homologada a una capacidad innata y espontánea, alejada sin duda de la movilización planificada del conjunto de conocimientos que el alumno posee en su memoria o en fuentes de información a su alcance. A la par corre implícita la concepción del texto literario, principalmente en su vertiente poética, como una construcción agramatical y aleatoria a causa, entre otras, de la discutible utilización de los modelos vanguardistas, del hecho de que cualquier cambio en las figuras retóricas o en la estructura del texto no sea sometida a las reglas de coherencia textual, o de la escasa atención concedida a la articulación lingüística en favor de un texto basado en la simple yuxtaposición acumulativa de elementos.

Por lo que respecta al ensayo de estructuras textuales, uno de los modelos más generalizados ha sido el de la morfología del cuento a partir de las definiciones de Propp, Todorov, o Greimas; esta aplicación escolar parece responder a la necesidad de técnicas de análisis percibidas como más científicas por los enseñantes, pero la dificultad de su abstracción conduce a su inevitable deformación y realiza una más que dudosa aportación a la gratificación lectora.

A pesar de estas reservas, es precisamente esta vía imitativa o de escritura con reglas la que, a la luz de las nuevas teorías, está ampliando con fuerza su justificación y rentabilidad escolar si se desarrolla a partir de una clara delimitación de objetivos, desde parámetros más controlados y con atención hacia la conservación de una raíz motivadora que no les convierta en una nueva retórica vacía de contenido.

\section{HACIA UNA EDUCACION LITERARIA}

Si la escuela debe garantizar el aprendizaje de la lectura escrita a todos los ciudadanos, probablemente sea fácil convenir que la lectura de textos literarios constituye una de las finalidades culturales de ese acceso. Aunque sin detenernos en este momento en exponer las reflexiones educativas más recientes (Armellini, 1987) sobre la actualidad de la importancia formativa de este encuentro entre el lector y la representación de la experiencia cultural a través de la palabra, podemos convenir en la necesidad de una educación literaria en la escuela básica que, desde el punto de vista de la recepción, debe concebirse como el desarrollo de las habilidades y competencias necesarias para la comprensión de la comunicación literaria.

En correspondencia con su integración en la formación lectora, la planificación de la intervención educativa sobre la lectura literaria debe tomar como punto de referencia las investigaciones realizadas durante los últimos años sobre la actividad lectora (qué es leer) y sobre qué tipo de ayudas facilitan la adquisición de esta capacidad (cómo enseñar a leer), investigaciones y avances de las que diversos autores (Ferreiro y Gómez Palacio, 1982; Alonso y Mateos, 1985; Colomer y Camps, 1991, etc.) han realizado síntesis especialmente útiles en el campo educativo. Si creemos que «la finalidad formativa 
de la educación literaria se puede resumir en la formación del lector competente", tal como afirma el proyecto para la educación literaria en Italia del Seminario della ricerca Dillis (1986), deberemos partir de la programación general de la enseñanza de la lectura para especificar èl tratamiento que debe otorgarse al texto literario.

Resulta operativo a este respecto partir de la consideración de la lectura como una actividad compuesta por dos tipos de conductas bien diferenciadas aunque simultáneas y complementarias: por una parte, un comportamiento lector que se dirige a la construcción del sentido a partir de actividades psicomotrices y de razonamiento, $\mathrm{y}$, por otra, un comportamiento lingǘstico que se adecua a las características de la lengua escrita. La capacidad de saber leer quedaría así definida, según Charmeux (1985), como la posibilidad de usar estos comportamientos como propios e incluiría diversas dimensiones afectivas (de implicación y seguridad), cognitivas (de formalización de los conocimientos implicados) y pragmáticas (de dominio y adaptabilidad de las conductas lectoras).

Estas coordenadas ofrecen la posibilidad de concretar y programar los aspectos implicados en la construcción de un saber leer literario por parte de los alumnos en el marco de la programación de su educación lectora en la escuela. Este saber puede esquematizarse en la adquisición de los siguientes componentes:

\section{A nivel de comportamiento lector}

\subsection{Desarrollo de la familiarización y seguridad respecto al texto literario}

Con la posibilidad consiguiente de adecuarse a esta situación de comunicación y a sus finalidades habituales de entrenamiento, placer, ampliación de la experiencia vital, etc., así como de adoptar las formas de lectura pertinentes. La escuela debería asegurar que todos los alumnos perciban la lectura literaria como una actividad que les compete personalmente, que puede formar parte de su mundo y en la que se mueven con soltura en su experiencia de cuándo, dónde, cómo y con qué intención se lee literatura en nuestra sociedad. Para ello resulta indispensable optar por una amplia creación de situaciones de auténtica lectura por placer que no conduzcan sistemáticamente al ejercicio escolar. En el establecimiento de este tipo de situaciones habrá que contemplar la distinción y relación entre las actividades de incitación a la lectura y las de formación de hábitos permanentes y habrá que incluir también la ampliación de las formas de lectura, reducidas a menudo a un mero consumismo argumental, con la inclusión de actitudes de implicación personal, de valoración estética y de distanciamiento crítico.

\subsection{Aprendizaje de la actividad de construcción del sentido del texto}

La consecución de una representación mental coherente y adecuada al texto leído debe constituir, precisamente, el núcleo de la intervención educativa en el terreno de la formación lectora. A nivel literario su desarrollo requiere:

A) Una selección de los textos propuestos concebida, no como una antología de grandes autores, sino como un recorrido en el aprendizaje de la co- 
municación literaria a partir del corpus que puede ser realmente comprendido por los alumnos.

B) La ponderación de los conocimientos previos del lector, ya que la capacidad de comprender un texto depende de la posibilidad de relacionar su mensaje con los esquemas conceptuales propios. La intervención educativa deberá dirigirse tanto a la adecuación de los textos como al establecimiento de los enlaces necesarios entre texto y lector. La representación mental que el lector de literatura debe formarse atañe al estado de desarrollo de su imaginario personal y a la confrontación entre su propia visión del mundo, y de él mismo, con la elaboración cultural de la experiencia humana que le ofrece cualquier obra literaria. Bajo este enfoque debería entenderse desde la escuela la posibilidad de contextualizar literaria y culturalmente el texto: como una vía para enriquecer el diálogo del lector con el texto al hacer posibles nuevas conexiones entre la representación obtenida y la representáción del mundo anterior a la lectura. Y esta misma perspectiva debería marcar el sentido y los límites de la información contextual ofrecida en la escuela obligatoria.

C) La sistematización de la ayuda necesaria para la adquisición de los mecanismos propios de todo acto de lectura: anticipar, comprobar y controlar la significación obtenida. Deberán proponerse actividades de anticipación a partir de una correcta selección de indicios (y saber qué indicios son relevantes en literatura), la ejercitación del razonamiento deductivo y por inferencia según las convenciones de los distintos géneros y el autocontrol continuado del lector sobre la coherencia de su representación mental según el baremo de la ficción literaria. La comparación y el debate sobre la interpretación obtenida por los distintos lectores permitirá constatar la pluralidad de lecturas consustancial al texto literario.

\section{A nivel de comportamiento lingüístico}

2.1. Desarrollo de la capacidad para caracterizar y situar el texto literario entre las variables lingüisticas que el dominio de la lengua pone a nuestra disposición. La familiarización y conocimiento de las formas organizativas del texto así como de los diversos géneros y modalidades del texto literario, permite activar los esquemas mentales adecuados y obtener una lectura comprensiva con mayor facilidad. Deberán tenerse en cuenta, pues, las características textuales que diferencian la literatura de los otros textos lingüísticos (sus diferencias en el funcionamiento de la coherencia pragmática, su violación de las reglas habituales de cohesión, su semantización de todos los niveles del texto, etc.) y sus formas tipificadas de organización.

2.2. Apropiación de aspectos especificos del texto literario como variable de las características linguísticas del texto escrito, con especial atención a la materialidad del texto literario como forma lingüística construída para su valoración en sí misma, y al uso especial de los recursos lingüísticos que ello conlleva. Es en este sentido que debe procederse, en primer lugar, a la selección de los conocimientos literarios realmente necesarios y convenientes para que los alumnos aumenten, no su información, sino su saber leer literario, $\mathrm{y}$, en segundo lugari a la programación de estos conocimientos a lo largo de la educación obligatoria teniendo en cuenta su posible división entre conocimientos implícitos o/y progresivamente explícitos.

En definitiva, la educación lectora nos proporciona la base para el desarrollo 


\section{Esquema de los componentes de la educación literaria}

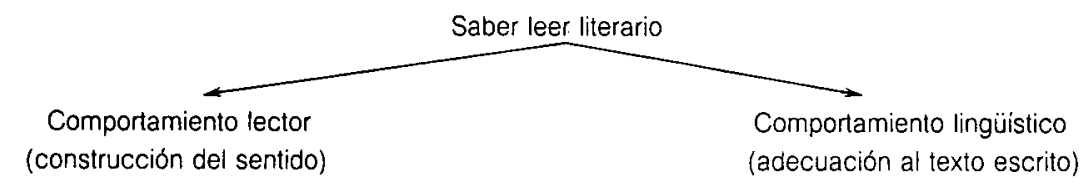

\section{Aproplarse el texto}

A través de actividades de

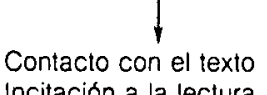

Incitación a la lectura

Formación de hábitos lectores

Ampliación formas de lectura

\section{Aprender a comprender}

a través de:

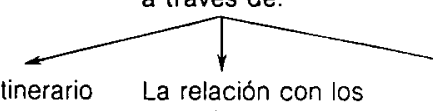

Un itinerario

de tecturas

comprensible

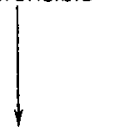

conocimientos previos

al lector.

La contextualización

literaria y cultura

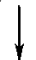

mecanismos lectores

de la pluralidad

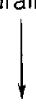

La aplicación de los

Distinguir el texto literario

y sus formas de organización

A través de:

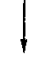

El contraste entre:

- textos diversos

- textos literarios

\section{Conocer sus rasgos}

especificos

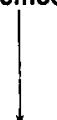

El aprendizaje de una selección adecuada de conocimientos sobre la construcción literaria

Situaciones, actividades y ejercicios de lectura ( $y$ escritura) literaria 
de una programación específica de la educación literaria. La concreción de este objetivo formativo debe contemplar una propuesta metodológica que genere un conjunto de nuevas prácticas educativas donde se vertebren y obtengan sentido los avances ya producidos en el marco escolar. Tal vez en estos momentos nos hallemos ya en condiciones de determinar el tipo y la articulación de las actividades escolares que pueden facilitar en mayor medida el aprendizaje de los alumnos en el contexto de una nueva situación de interacción educativa que les otorgue una gradual autonomía en la construcción de su saber leer literario.

\section{Esquema de los cambios producidos en el modelo tradicional de enseñanza literaria}

Primacía del registro literario en la enseñanza de la lengua

Enseñanza como transmisión la interpretación de los textos por parte del profesor. Prioridad de la memorización

Lectura de textos de autores clásicos

Programación histórica de los contenidos. Más información que lectura de texto

Expresión escrita limitada a la paráfrasis, el comentario y el resumen
Integración del registro literario en los diversos usos lingüísticos

Construcción del conocimiento por parte del alumno. Interacción educativa. Prioridad de la comprensión

\section{Ampliación del corpus literario}

Diversidad en los ejes de contenidos (programación por géneros, técnicas, etc.) Gran incremento del contacto con el texto

Amplia interrelación entre recepción literaria y expresión escrita

\section{Referencias}

Adam, J. M. (1987). Type de séquences élémentairess. En Pratiques, 56.

ADAm, J. M. (1988). Linguistique et littérature: qu'est-ce qu'un texte? En Littérature et enseignement, la perspective du lecteur, op. cit.

Alonso, J. y Mateos, M. del M. (1985). Comprensión lectora: modelos, entrenamientos, evolución. En Infancia y Aprendizaje, 31-32, 5-19.

ARMeLlini, G. (1987). Come e perché insegnare letteratura. Strategie e tattiche per la scuola secondaria. Bologna: Zanichelli.

BetTel heim, B. y ZeLAN, K. (1981). On learning to read. The child's fascination with meaning, Nueva York: Alfred A. Knopf (Trad. cast. Aprender a leer. Barcelona: Crítica, 1982).

Charmeux, É. (1985). Savoir lire au college. París: CEDIC.

Collins, A. y SMITH, E. (1980). Teaching the process of reading comprehension (Inf. Tec. n. ${ }^{\circ}$ 182). Urbana Univ. Illinois, Center for Study of Reading.

Colomer, T. y CAMPS, A. (1991). Ensenyar a llegir, ensenyar a comprendre. Barcelona: Ed. 62-Rosa Sensat.

COMBETTES, B. (1988). Linguistique et enseignement de fraçais langue maternalle: tendances nouvelles. En Études de Linguistique Appliquée 72.

Coveri, L. (ac.di) (1986). Insegnare letterature nell scuola superiores. Firenze: La Nuova Italia.

FEDericis, L. DE (1985). Il debattito sull'insegnemanto di letteratura. En A. Colombo y C. Sommadossi (Ed) LEND Educazione letteraria. Milan: Edizioni Scolastiche Bruno Mondadori. 
IFRRFIRO, E. y G(mil:\% PAI.Ac:Io, M. (comp.) (1982). Nuevas perspectivas sobre los procesos de' lectura y escritura. Madrid: Siglo XXI.

Littérature et enseignement, la perspective du lecteur (1988) n." spécial Le français dans le monde, recherches et aplications.

I.uginiNi, E. (acdi) (1985). Insegnare letterature nella scuola dellobbligo. Iirenze: La Nuova Italia.

Plamillan, A. (1982). Pratiques d'écriture. París: Cediz-Nathan.

P1.TlJtan, A. (1984). De la lecture è l'écriture: la transformation de texte. París: Cedic-Nathan.

Siminakio Di.i.a Ricirca Dil.ss (1986). Centro Europeo della Fiducazione, Villa Falconieri, Frascati (Roma).

Sulf, I. (1987). L'ensenyament de la comprensió lectora. Barcelona: CEAC.

WII.I.S, G. (1986). The meaning makers. Heinemanu Educational Books (Trad. cast. Aprender a leer y a escribir. Barcelona: Laia, 1988).

\section{De la enseñanza de la literatura a la educación literaria. M. T. Colomer CLEE, 1991, 9, pp. 18-31}

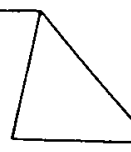

Resumen: Este artículo señala que los avances producidos en las últimas décadas en la educación lingüistica ban propiciado diversos cambios en la enseñanza de la literatura, pero no se han traducido aún en un planteamiento global sobre nuevas bases teóricas. Destaca las prácticas de enseñanza más relevantes en estos momentos y propone su articulación e integración en un nuevo marco general de educación lectora.

Datos sobre la autora: Teresa Colomer es profesora de Didáctica de la lengua y la literatura en la Escuela de Formación del profesorado de EGB de la Universidad Autónoma de Barcelona. Su campo de trabajo se centra en la didáctica de la literatura y en la literatura infantil y juvenil. Es coordinadora de un curso de postgrado sobre Biblioteca escolar.

Dirección: Universidad Autónoma de Barcelona. Escola Universitaria de Mestres "Sant Cugat», Edifici G, 08193 Barcelona.

( ) de todos los artículos. Deberá solicitarse por escrito autorización de CL\&E para el uso en forma de facsímil, fotocopia o cualquiẹ otro medio de reproducción. CL\&E se reserva el derecho de interponer las acciones legales necesarias en aquellos casos en que se contravenga la ley de derechos de autor. 